\title{
Effect of external electric field on the surface energetics of $\mathrm{Ag} / \mathrm{Si}(111)$
}

\author{
Y. He, ${ }^{1}$ X. Y. Wei, ${ }^{1}$ C. T. Chan, ${ }^{2}$ and J. G. Che ${ }^{1, *}$ \\ ${ }^{1}$ Surface Physics Laboratory, Fudan University, Shanghai 200433, People's Republic of China \\ ${ }^{2}$ Department of Physics, Hong Kong University of Science and Technology, Hong Kong, People's Republic of China
}

(Received 24 June 2004; published 4 January 2005)

\begin{abstract}
Based on first-principles calculations, we found that the surface adsorption energetics of $\mathrm{Ag}$ on a $\mathrm{Si}(111)$ surface can be modified by imposing an external electric field. The preferred adsorption site is found to be field dependent. The most energetically favorable adsorption site of $\mathrm{Ag}$ on the $\mathrm{Si}(111)$ surface, among all the considered sites (hcp, fcc, bridge, and top), will undergo a transformation from the fcc site to the top site with an increase in field strength when the surface is positively biased.
\end{abstract}

DOI: 10.1103/PhysRevB.71.045401

PACS number(s): 68.35.Fx, 68.37.Ef, 68.43.Bc, 68.43.Jk

Surface diffusion and surface growth processes are influenced by various factors such as deposition rate, substrate temperature, surface reconstruction, and external stress. ${ }^{1,2}$ It is always desirable to have additional control over such processes to attain certain metastable or stable surface configurations that are difficult or impossible to obtain with conventional procedures. The use of surfactants to achieve layer-bylayer growth ${ }^{3-5}$ is a good example. However, surfactants may contaminate the surface and are thus of limited use in technology. Physical effects such as external fields may be advantageous since the surface remains "clean" when the external field is removed.

There has been some evidence that surface morphology is strongly affected by an imposed electric field. It is well documented that potential-induced surface morphological changes are observed in metal/electrolyte interfaces. ${ }^{6}$ An excess surface charge can induce a missing-row reconstruction on $\operatorname{Ag}(110) .^{7}$ Adatoms on the surface are stabilized by the presence of the tip and energy barriers for the diffusion processes in the region of the scanning tunneling microscopy(STM) tip. ${ }^{8}$ The presence of an electrostatic field is found to dramatically enhance the kinetics of $\mathrm{Al}(111)$ oxidation when $\mathrm{O}_{2}$ molecules interact with the charged surface after electron bombardment. ${ }^{9}$ It was also found that the electric field or surface charging can stabilize surface vacancy arrays in metal bcc(100) surfaces, leading to a change of surface configurations. ${ }^{10}$ The field effects on $\mathrm{Si}$ ad-dimer diffusion on $\mathrm{Si}(001)$ have been shown by experimental ${ }^{11}$ and theoretical $^{12}$ investigations. The electric field effect on surface diffusion has been investigated by the field ion microscopy (FIM) technique. Kellogg has found that an external electric field can indeed inhibit or promote surface selfdiffusion on $\mathrm{Pt}(001)$ surface, ${ }^{13}$ and Feibelman tried to explain this experimental observation using $a b$ initio calculations. ${ }^{14}$

In this work, we will demonstrate that an externally imposed electric field may indeed modify or control surface adsorption energetics. Because of its importance for a wide variety of surface phenomena (STM, FIM, electrochemical interfaces), understanding the influence of an electric field on surface properties is also essential for interpreting some experimental results.

Our main finding is that the binding energy of $\mathrm{Ag}$ on various sites of the $\mathrm{Si}(111)$ substrate could be influenced by an external electric field applied to the surfaces. For the case of zero electric field, the adsorbate $\mathrm{Ag}$ on $\mathrm{Si}(111)$ has the lowest energy at the fcc site and the highest energy at the top site among all the sites considered (fcc, hcp, bridge, and top). If an external electric field is applied to the system, the relative binding energies will be changed accordingly. At about $1.2 \mathrm{~V} / \AA$, the binding energy of fcc and top sites are almost the same, and the top site becomes the energetically favored site for Ag when the external electric field becomes larger than $1.2 \mathrm{~V} / \AA$. This means that the preferred site of adsorption, the surface barriers, and the diffusion pathways can be varied and thus surface energetics and kinetics can be influenced by applying an external electric field on surfaces.

These results were obtained by first-principles calculations based on density functional theory (DFT) as implemented in the VASP package. ${ }^{15}$ The wave functions are expanded in a plane-wave basis set, the electron-ion interactions are described by ultrasoft pseudopotentials, and the generalized gradient approximation for the exchangecorrelation functional has been used. We used a $p(3 \times 3)$ surface unit cell that contains 14 atomic layers with $126 \mathrm{Si}$ atoms and $2 \mathrm{Ag}$ adatoms (one on each side of the slab). The $k$ points (4 points in the irreducible surface Brillouin zone), slab thickness (14 layers), energy cutoff $(250 \mathrm{eV})$, and vacuum spacing (22 $\AA$ ) are all chosen to ensure convergence to $0.03 \mathrm{eV}$ per adatom. To include the effect of an external electric field, the external uniform charge sheets are placed in the vacuum on either side of the slab. ${ }^{10,16}$ Only the atoms in the two center layers of the slab are kept fixed, and the $z$ coordinate of the adatom and all coordinates of the substrate atoms are relaxed during the structural relaxation.

We choose $\mathrm{Ag} / \mathrm{Si}(111)$ as a prototype system because it is an important system that has been the subject of intensive studies for a long time. We consider the energetics of $\mathrm{Ag}$ adsorption on an ideal $\mathrm{Si}(111)(1 \times 1)$ surface. A unit cell of $p(3 \times 3)$ was chosen to minimize interaction between $\mathrm{Ag}$ adatoms. Four important adsorption sites (top, fcc, hcp, and bridge) on $\mathrm{Si}(111)$ surface are considered, which are shown in Fig. 1. These adsorption sites contain one-, two-, and threefold symmetry and are used to investigate effects of electric field on Ag adatom on Si(111) surface. The one-, two-, and threefold symmetries can represent important features of adsorption symmetry on more complex reconstruc- 


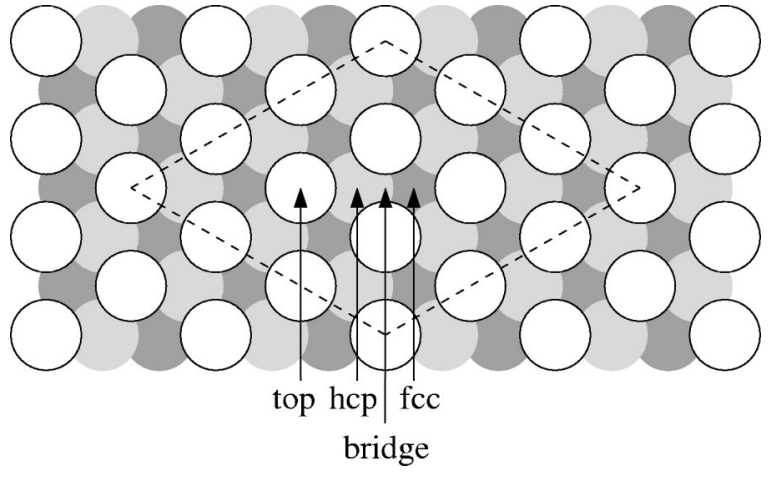

FIG. 1. The structures considered for Ag adsorbed (fcc, hcp, top, and bridge site) on the $\mathrm{Si}(111)$ surface, at a coverage of one-ninth of a monolayer, with a $(3 \times 3)$ unit cell.

tion on the $\mathrm{Si}(111)$ surface. We will see that electric field affects mainly the bonds between $\mathrm{Ag}$ and the $\mathrm{Si}$ atoms beneath.

The atomic geometries of $\mathrm{Ag}$ on the $\operatorname{Si}(111) p(3 \times 3)$ surface are optimized by calculating the forces and total energies for these sites. The calculated results of the adsorption energy differences for these sites are listed in Table I with the adsorption energy of the fcc site as the reference. The most stable adsorption site in the absence of an external electric field is the fcc site. It is $0.54 \mathrm{eV} /$ (adatom) lower in energy than the top site, which has the highest energy among all the sites considered. For Ag on the $\mathrm{Si}(111)$ surface, the fcc, hcp, and bridge sites have nearly the same energies. It is consistent with the fact that the large atomic radius of the silver atom leads to a relatively large adsorbate-substrate distance and thus the Ag adsorbates will experience a relatively small substrate electron density corrugation. The fcc site is always slightly favored over the hep site in energy in our calculations.

We then applied an electric field to the surface and calculated the adsorption energies for various sites as a function of the electric field strength. The results are shown in Table I. We plot the energy difference between the adsorption of $\mathrm{Ag}$ on the fcc and top sites as a function of field strength in Fig. 2. A positive field means that the surface is positively biased. The difference in adsorption energy decreases monotonically with increase of the field strength in the range we have considered. We see that the most energetically favorable adsorption site changes from fcc to top with the increase of the field

TABLE I. The energy differences (in $\mathrm{eV}$ ) between different sites for different strength of electric field (in V/ $\AA$ ). The energy in the fcc site is used as the reference.

\begin{tabular}{cccc}
\hline \hline$E$ field & Top & Bridge & Hcp \\
\hline 0.0 & 0.54 & 0.02 & 0.08 \\
0.5 & 0.27 & 0.05 & 0.08 \\
1.0 & 0.07 & 0.06 & 0.08 \\
1.2 & -0.03 & 0.07 & 0.08 \\
1.5 & -0.18 & 0.07 & 0.08 \\
2.0 & -0.49 & 0.02 & 0.07 \\
\hline \hline
\end{tabular}

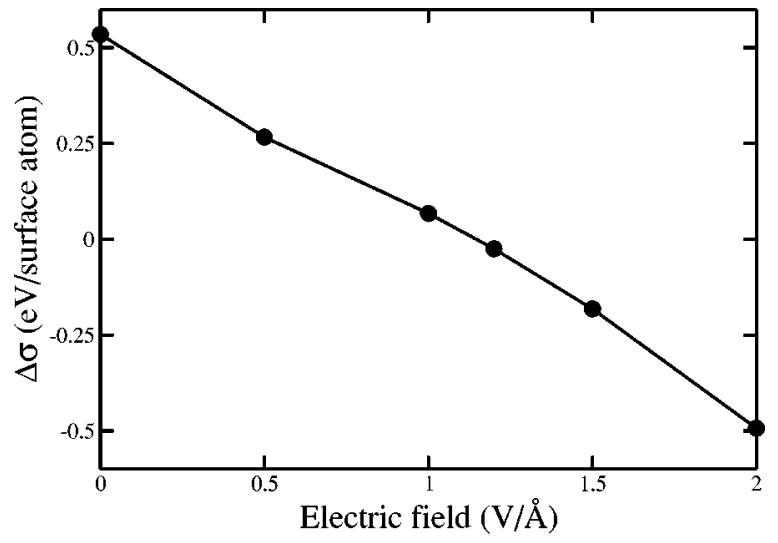

FIG. 2. Energy difference between top and fcc sites in the presence of an external electric field.

strength: When the field reaches about $1.2 \mathrm{~V} / \AA$, the difference of the adsorption energy of $\mathrm{Ag}$ on $\mathrm{Si}(111)$ between the fcc and top site is almost zero. The top site becomes the most favorable site for $\mathrm{Ag}$ adsorption on the $\mathrm{Si}(111)$ surface with further increase of the field. As the field strength reaches $2.0 \mathrm{~V} / \AA$, the energy difference between top and fcc is $-0.49 \mathrm{eV} /$ (adatom).

The adsorption energies of all four sites are nearly the same when the field strength reaches about $1.2 \mathrm{~V} / \AA$. At higher field strengths, the top site becomes the favored site, and the $\mathrm{Ag}$ atoms will be pinned there. Our results suggest that with increasing field strength or surface charging, the surface diffusion coefficient of $\mathrm{Ag}$ adatoms on the $\mathrm{Si}(111)$ surface rapidly decreases due to pinning at the top sites.

Now we turn to the origin of the field effects. We first calculate the surface dipoles for the top and the fcc sites at zero field by a direct integration of the charge densities starting from the center of the slab into the middle of the vacuum region. ${ }^{17}$ We found that the surface dipoles always points inwards at $\mathbf{E}=\mathbf{0}$ (and in the following, an inward surface dipole will be called a negative dipole), and the magnitude of surface dipole at the fcc site is larger than that of the top site by a value of $0.085 e \AA$ per $\mathrm{Ag}$ atom. Such an intrinsic difference in surface dipole will contribute to a difference in energy when there is an external field. Since the intrinsic surface dipole is antiparallel to the external field that points outwards, the site with a small dipole will be favored. That means that the intrinsic dipole is going to favor the top site over the fcc site by a value of $0.085 E$, with $E$ measured in $\mathrm{V} / \AA$. While this is qualitatively consistent with the observed trend that the top site is favored in a strong positive field, we note that the magnitude is much smaller than the calculated energy difference. Total energy calculations show that the energy difference between the top and fcc site changes from $0.54 \mathrm{eV}$ at zero field to $0.07 \mathrm{eV}$ at $E=1 \mathrm{~V} / \AA$, or a change of $0.47 \mathrm{eV}$, while at $E=1 \mathrm{~V} / \AA$, the intrinsic surface dipoles contributes only $0.085 \mathrm{eV}$.

In Fig. 3, we show the adsorption energy of $\mathrm{Ag}$ at various sites as a function of the external field strength. The adsorption energy is defined as $E_{\text {system with field }}-E_{\mathrm{Si} \text { surface with field }}$ $-E_{\mathrm{Ag} \text { bulk }}$, where $E_{\text {system with field }}$ is the total energy of the system with $\mathrm{Ag}$ atoms at the respective adsorption sites and $E$ 


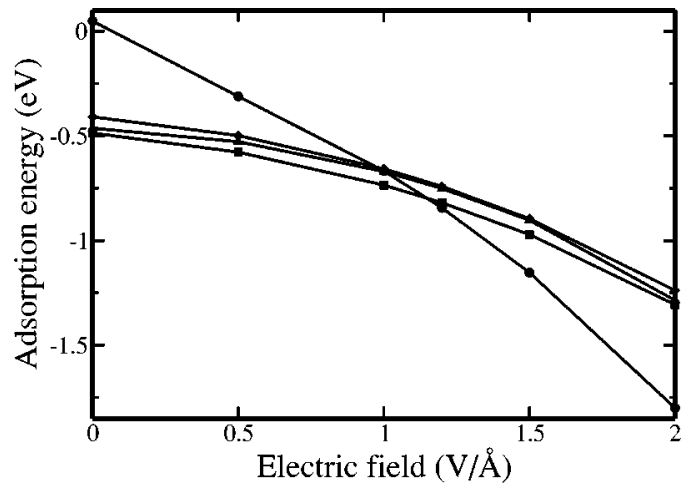

FIG. 3. Adsorption energy as a function of the external field strength. Circles, squares, diamonds, and up triangles refer to adsorption energies for the top, fcc, hcp, and bridge site, respectively.

field imposed, and $E_{\mathrm{Si}}$ surface with field is the total energy of the $\mathrm{Si}$ slab with the same external field imposed. The unit cells in both cases are of exactly the same size. $E_{\mathrm{Ag} \text { bulk }}$ is the energy of $\mathrm{Ag}$ in the bulk without considering field. We note that at zero fields, all sites have negative adsorption energy except at the lowest coordinated top site, which means that Ag absorbs exothermally on Si surface. The absorption energy increases with the $E$ field, and the increase is much faster than linear, indicating that the effect must be due to the induced dipole effect rather than intrinsic dipole effect.

We next consider the field-induced effect. It is natural that an external field will induce charge rearrangement, and the overall screening charge should tend to shield the bulk from the external field. We thus expect that the screening charge has an overall positive sign (depletion of electrons) from the surface. The sign of the induced dipole should be positive (points outward), and we found numerically that it is indeed the case. We note that the absolute magnitude of the induced dipole depends on the origin of charge integration because there is an excess of screening charge on the surface. However, the difference in the induced dipole between two sites is unambiguous. We found that as long as we integrate the charge from the middle of the slab, the induced dipole is always positive and the magnitude for the top site is always larger than that of the fcc site. Since the induced dipole always lowers the energy of the system, this means that the induced dipole is going to further favor the top site over the fcc site. At a field of $E=1 \mathrm{~V} / \AA$, the difference in surface dipole between the top and fcc site (intrinsic and induced together) is $0.45 e \AA$, which is consistent with the energy difference of $0.47 \mathrm{eV}$ mentioned above. In fact, the agreement at $E=1 \mathrm{~V} / \AA$ is better than we can expect because the total field is not uniform at the Ag site and there will be some changes in energy due to changes in bonding configurations manifested in the change of exchange-correlation energies as the charge density distribution are different.

In order to examine the field effect on charge and bonding, a pseudocharge difference is plotted in Fig. 4. It is obtained by subtracting the charge obtained in two separate calculations: one with and the other without an external electric field of $2.0 \mathrm{~V} / \AA$. The charge density difference for other field strengths are qualitatively the same. The atomic posi- (a)
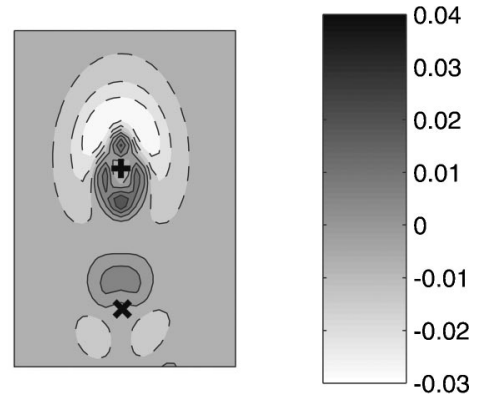

(b)
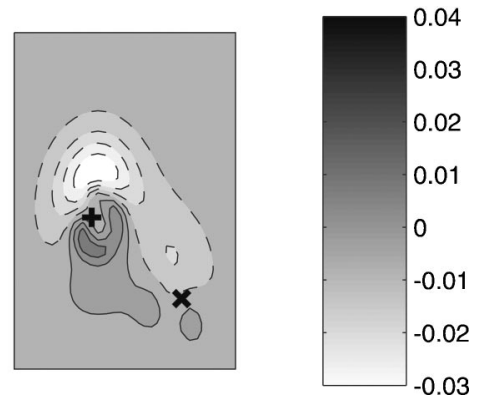

FIG. 4. Charge density difference $\left(e / \AA^{3}\right)$ plots for top (a) and fcc (b) adsorption sites at a field of $2 \mathrm{~V} / \AA$.

tions remain the same in the both calculations and correspond to those that minimize the total energy under the external electric field. Figures 4(a) and 4(b) show the charge difference under an electric field of $2.0 \mathrm{~V} / \AA$ in the (110) plane for the case of $\mathrm{Ag}$ on the top site and on the fcc site of $\mathrm{Si}(111)$ surface, respectively. In Fig. 4 the region encompassed by dashed lines indicates a depletion of electrons, while the region encompassed by solid lines means an excess of electrons. The symbols + and $\times$ in the figure indicate positions of $\mathrm{Ag}$ and $\mathrm{Si}$, respectively.

We see from Fig. 4 that there is a cloud of missing electrons over the Ag atom in the top site, which serves to screen the external field. For Ag in the fcc site, the Si atoms bonded directly to the $\mathrm{Ag}$ atoms also have the cloud of screening charge. This cloud of screening charge contributes to the positive surface dipole. As Fig. 4 shows, the Ag-Si bond is vertical on the top site and parallel to the external field, leading to a stronger polarizability and maximum field-dipole interaction. For $\mathrm{Ag}$ in the fcc site, the Ag-Si bond is at an angle of $41^{\circ}$, leading to a weaker field-induced effect. In addition, we observe from Fig. 4(a) that more electronic charge is redistributed to the bonding region between $\mathrm{Ag}$ and $\mathrm{Si}$, consistent with an enhanced bond strength, while the effect is less conspicuous for the fcc site.

Up to now, our discussions are constrained in $\mathrm{Ag}$ on $\mathrm{Si}(111)(1 \times 1)$. The clean $\mathrm{Si}(111)$ surface shows very complex reconstructions, depending on its preparation and annealing techniques: ${ }^{18}$ a $(2 \times 1)$ reconstruction below $603 \mathrm{~K}$, a $(7 \times 7)$ reconstruction annealing upon $873 \mathrm{~K}$. The main conclusion of this calculation is the relative binding energy for $\mathrm{Ag}$ on $\mathrm{Si}$ is field dependent, mainly because of the different strength of the induced dipole moment at different sites, which depends on the induced charge that is in turn dictated by coordination and geometry of the local Ag-Si bonding. 
From this point of view, our conclusions could be qualitatively extended to more complex surface structures. Our conclusion that the lowest coordinated site is favored in high positive field should be qualtitatively correct in more complex Si(111) surfaces. However, the screening properties of the surface does depend on the details of the surface construction. This means that the quantitative results will depend on how the surface is reconstructed.

In summary, our calculations found that the adsorption of Ag on $\mathrm{Si}(111)$ surface has the lowest energy at the fcc site at zero field. As the field strength reaches $1.2 \mathrm{~V} / \AA$, the most stable site of adsorption Ag switches to the top site. At higher field strength, the diffusion of silver adatoms will be restrained greatly and the Ag adatoms are pinned near the top site. The lower coordinated top site is favored in a strong positive field since it has a stronger induced dipole than the higher coordinated sites.

This work was supported by the National Natural Science Foundation of China. We are grateful to the Large Scale Scientific Computation Research Project for computer time.
*Author to whom correspondence should be addressed.

${ }^{1}$ Z. Y. Zhang and M. G. Lagally, Science 276, 377 (1997).

${ }^{2}$ C. Ratsch, A. P. Seitsonen, and M. Scheffler, Phys. Rev. B 55, 6750 (1997); E. Penev, P. Kratzer, and M. Scheffler, ibid. 64, 085401 (2001).

${ }^{3}$ M. Copel, M. C. Reuter, E. Kaxiras, and R. M. Tromp, Phys. Rev. Lett. 63, 632 (1989).

${ }^{4}$ M. Horn-von Hoegen, F. K. LeGoues, M. Copel, M. C. Reuter, and R. M. Tromp, Phys. Rev. Lett. 67, 1130 (1991); N. Grandjean, J. Massies, and V. H. Etgens, ibid. 69, 796 (1992).

${ }^{5}$ H. A. van der Vegt, H. M. van Pinxteren, M. Lohmeier, E. Vlieg, and J. M. C. Thornton, Phys. Rev. Lett. 68, 333 (1992).

${ }^{6}$ J. Wang, A. J. Davenport, H. S. Isaacs, and B. M. Ocko, Science 255, 1416 (1992); D. M. Kolb, Prog. Surf. Sci. 51, 109 (1996).

${ }^{7}$ C. L. Fu and K. M. Ho, Phys. Rev. Lett. 63, 1617 (1989).

${ }^{8}$ M. R. Sørensen, K. W. Jacobsen, and H. Jónsson, Phys. Rev. Lett. 77, 5067 (1996); B. S. Swartzentruber, A. P. Smith, and H. Jónsson, ibid. 77, 2518 (1996).

${ }^{9}$ I. Popova, V. Zhukov, and J. T. Yates, Jr., Phys. Rev. Lett. 89,
276101 (2002).

${ }^{10}$ J. G. Che, Z. Z. Zhu, and C. T. Chan, Phys. Rev. Lett. 82, 3292 (1999), and references therein.

${ }^{11}$ J. M. Carpinelli and B. S. Swartzentruber, Phys. Rev. B 58, R13423 (1998).

${ }^{12}$ T. R. Mattsson, B. S. Swartzentruber, R. Stumpf, and P. J. Feibelman, Surf. Sci. 536, 121 (2003).

${ }^{13}$ G. L. Kellogg, Phys. Rev. Lett. 70, 1631 (1993).

${ }^{14}$ P. J. Feibelman, Phys. Rev. B 64, 125403 (2001).

${ }^{15}$ G. Kresse and J. Hafner, Phys. Rev. B 47, 558 (1993); G. Kresse and J. Hafner, ibid. 49, 14251 (1994); G. Kresse and J. Furthmüller, Comput. Mater. Sci. 6, 15 (1996); Phys. Rev. B 54, 11169 (1996).

${ }^{16}$ J. G. Che and C. T. Chan, Phys. Rev. B 67, 125411 (2003).

${ }^{17}$ T. C. Leung, C. L. Kao, W. S. Su, Y. J. Feng, and C. T. Chan, Phys. Rev. B 68, 195408 (2003).

${ }^{18}$ W. Mönch, Semiconductor Surfaces and Interfaces, 2nd ed., Springer Series in Surface Science (Springer-Verlag, Berlin, 1995), Vol. 26. 